\title{
Improved glucose tolerance in mice receiving intraperitoneal transplantation of normal fat tissue
}

\author{
D. Konrad • A. Rudich • E. J. Schoenle
}

Received: 27 November 2006 / Accepted: 20 December 2006/ Published online: 16 February 2007

(C) Springer-Verlag 2007

\begin{abstract}
Aims/hypothesis The association between increased (visceral) fat mass, insulin resistance and type 2 diabetes mellitus is well known. Yet, it is unclear whether the mere increase in intra-abdominal fat mass, or rather functional alterations in fat tissue in obesity contribute to the development of insulin resistance in obese patients. Here we attempted to isolate the metabolic effect of increased fat mass by fat tissue transplantation.

Methods Epididymal fat pads were removed from male C57B16/J mice and transplanted intraperitoneally into male littermates (recipients), increasing the combined perigonadal fat mass by $50 \%(p<0.005)$. At 4 and 8 weeks post-transplantation, glucose and insulin tolerance tests were performed, and insulin, NEFA and adipokines measured.

Results Circulating levels of NEFA, adiponectin and leptin were not significantly different between transplanted and sham-operated control mice, while results of the postprandial insulin tolerance test were similar between the two groups. In contrast, under fasting conditions, the mere increase in intra-abdominal fat mass resulted in decreased plasma glucose levels $(6.9 \pm 0.4$ vs $8.1 \pm 0.3 \mathrm{mmol} / \mathrm{l}$,
\end{abstract}

D. Konrad $(\bowtie) \cdot$ E. J. Schoenle

Department of Endocrinology and Diabetology,

University Children's Hospital,

Steinwiesstrasse 75 ,

CH-8032 Zurich, Switzerland

e-mail: daniel.konrad@kispi.unizh.ch

A. Rudich

Department of Clinical Biochemistry, Ben-Gurion University, Beer-Sheva, Israel

A. Rudich

S. Daniel Centre for Health and Nutrition, Ben-Gurion University,

Beer-Sheva, Israel $p=0.03)$ and a $\sim 20 \%$ lower AUC in the glucose tolerance test $(p=0.02)$ in transplanted mice. Homeostasis model assessment of insulin resistance (HOMA-IR) was $4.1 \pm 0.4$ in transplanted mice (vs $6.2 \pm 0.7$ in sham-operated controls) $(p=0.02)$, suggesting improved insulin sensitivity. Linear regression modelling revealed that while total body weight positively correlated, as expected, with HOMA-IR ( $\beta$ : $0.728, p=0.006)$, higher transplanted fat mass correlated with lower HOMA-IR $(\beta$ : $-0.505, p=0.031)$.

Conclusions/interpretation Increasing intra-abdominal fat mass by transplantation of fat from normal mice improved, rather than impaired, fasting glucose tolerance and insulin sensitivity, achieving an effect opposite to the expected metabolic consequence of increased visceral fat in obesity.

Keywords Fat transplantation - Glucose homeostasis . Insulin resistance $\cdot$ Insulin sensitivity Obesity . Visceral adiposity

\begin{tabular}{|c|c|}
\hline Abbreviati & \\
\hline HOMA-IR & $\begin{array}{l}\text { homeostasis model assessment of insulin } \\
\text { resistance }\end{array}$ \\
\hline $\begin{array}{l}\text { ipGTT } \\
\text { ipITT }\end{array}$ & $\begin{array}{l}\text { intraperitoneal glucose tolerance test } \\
\text { intraperitoneal insulin tolerance test }\end{array}$ \\
\hline
\end{tabular}

Introduction

Obesity is caused by energy intake in excess of energy expenditure, and is associated with an increased number and/or size of white adipocytes, which store the excessive energy in the form of triacylglycerol. The association between increased fat mass, insulin resistance and type 2 
diabetes mellitus is well known. Yet, regional body fat distribution may influence the occurrence of insulin resistance associated with obesity. Increased accumulation of visceral (intra-abdominal) fat seems to be particularly associated with an increased risk of insulin resistance and type 2 diabetes. Two major lines of observations suggest a causative role for intra-abdominal fat in this association. First, subjects with normal body weight, but with increased visceral adiposity (e.g. elderly people or patients with certain forms of lipodystrophy) are prone to develop insulin resistance $[1,2]$. Second, removal of intra-abdominal fat improved insulin sensitivity [3, 4], whereas the removal of subcutaneous fat tissue by liposuction in obese humans did not improve insulin action [5]. Thus, the pattern of body fat distribution, especially abdominal fat accumulation, determines insulin sensitivity.

One remaining question to define in this process is the relative contributions of: (1) the mere increase in intraabdominal fat mass; and (2) the role of alterations in fat tissue biology that accompany obesity. Increase in fat mass as such has been suggested to increase the delivery of non-esterified fatty acids through the portal circulation, resulting in increased hepatic and muscular triacylglycerol accumulation [6]. Similarly, altered adipose tissue biology can change inter-organ cross-talk mechanisms through alterations in its metabolic and/or endocrine function. For example, a recent study demonstrated that the degree of macrophage infiltration into omental fat of obese patients correlated with histopathological findings in liver biopsies [7].

To investigate the isolated role of a mere increase in intra-abdominal fat in mice, we used intra-abdominal fat transplantation to increase the intra-abdominal mass of 'normal' fat. We reasoned that if the mere increase in fat mass is the predominant process occurring in obesity, increasing intra-abdominal fat mass by surgical transplantation of fat tissue from a normal, non-obese littermate would induce metabolic consequences in the recipient mice that resemble those occurring in obesity. If, on the other hand, the predominating effect is obesity-induced alterations in the endocrine function of fat tissue, the addition of such fat mass would not impair metabolic regulation.

\section{Materials and methods}

Animals Male C57BL6JOlaHsd mice were purchased from Harlan Netherlands (Horst, the Netherlands). All mice were housed in a pathogen-free environment on a 12-h light-dark cycle, with free access to standard rodent diet (Provimi Kliba, Kaiseraugst, Switzerland). All protocols conformed to the Swiss animal protection laws and were approved by the Cantonal Veterinary Office in Zurich, Switzerland.
Surgical procedure Transplantation procedure was performed at 6 weeks of age. Mice were anaesthetised with isoflurane (Abbott, Baar, Switzerland). Both epididymal fat pads were removed from the donor mouse, rinsed with $0.9 \%$ saline and thereafter stitched to the visceral side of the peritoneum of the recipient mouse using Vicryl 5.0 (Johnson-Johnson, Spreitenbach, Switzerland). Except for a small piece of fat tissue fixed in $4 \%$ buffered formalin, the entire two fat pads were weighed and then transplanted. Sham-operated control mice received the same treatment, but instead of the fat pad transplantation, an artificial suture was performed with Vicryl. Subcutaneous injection of buprenorphine every $12 \mathrm{~h}$ for 2 days was used for analgesia. In order to prevent transplant rejection, donor and recipient mice were littermates.

Glucose and insulin tolerance tests For fasting intraperitoneal glucose tolerance test (ipGTT), either prolonged fasting (overnight, i.e. $12 \mathrm{~h}$ food withdrawal) or $7 \mathrm{~h}$ food withdrawal were used, as indicated. For intraperitoneal insulin tolerance test (ipITT) in the post-absorptive state, mice were fed and then fasted for $3 \mathrm{~h}$. Either glucose ( $2 \mathrm{~g} / \mathrm{kg}$ body weight) or human normal insulin $(0.75 \mathrm{U} / \mathrm{kg}$ body weight $)$ were injected intraperitoneally and blood was collected from the tail vein at different time points, as indicated [8]. Plasma glucose was measured using a glucose meter (Ascensia Contour; Bayer, Zurich, Switzerland).

Determination of plasma insulin, adipokines and NEFA levels Plasma was collected from the tail vein after $7 \mathrm{~h}$ of fasting. Insulin was determined using an ELISA kit (Ultra Sensitive Rat Insulin ELISA; Crystal Chem, Downers Grove, IL, USA). NEFA levels were measured using the ACS-ACOD-MEHA method (Wako Chemicals GmbH, Neuss, Germany). Plasma leptin and adiponectin were determined with commercially available mouse endocrine and single plex adiponectin kits (MENDO-75K and MADPK-71K-ADPN; Linco Research, distributed by Labodia, Yens, Switzerland) that are capable of simultaneously measuring different adipokines in mouse serum or plasma. The two kits are based on Luminex xMAP technology (Linco Research, St Charles, MI, USA) and use microsphere bead sets that are uniquely labelled with a mixture of two fluorescent dyes. The captured antibodies specific for each analyte are covalently coupled to individual bead sets. At the time of the assay, a mixture of beads is incubated overnight at $4^{\circ} \mathrm{C}$ with $10 \mu \mathrm{l}$ of standards or mouse plasma samples in a 96-well filterbottom plate. On the next day, the beads are washed, and biotinylated detection antibody cocktail is added and incubated for $30 \mathrm{~min}$ at room temperature, followed by the addition of streptavidin-phycoerythrin and incubation for another $30 \mathrm{~min}$. After a final wash, the resuspended 
beads are read on a Luminex 100 reader (Bio-Rad Laboratories, Hercules, CA, USA) and the concentration of each analyte in the samples to be tested is determined on the basis of individual standard curves.

Determination of homeostasis model assessment of insulin resistance The homeostasis model assessment of insulin resistance (HOMA-IR) was calculated using glucose and insulin determinations obtained after $7 \mathrm{~h}$ of food withdrawal, using the following formula: fasting glucose $(\mathrm{mmol} / \mathrm{l}) \times$ fasting insulin $(\mathrm{mU} / \mathrm{ml}) / 22.5[9,10]$.

Histology Epididymal fat tissues were fixed in 4\% buffered formalin and imbedded in paraffin. Sections were obtained either at the time of transplantation or 8 weeks after transplantation and stained with haematoxylin-eosin. For each mouse at least four fields (representing a total of 350 to 550 adipocytes) were analysed. Images were analysed using ImageJ software for quantification (National Institutes of Health, Bethesda, MD, USA).

Data analysis Statistical analyses of the obtained data were performed using Student's $t$ test or ANOVA (Fisher's multiple comparisons test), as indicated. We performed Pearson correlations between the continuous variables and estimated the independent effect of fat transplantation by further adjustment for total body weight using a linear regression model (SPSS package software, version 14).

\section{Results}

Intraperitoneal fat transplantation increases intra-abdominal fat mass without significantly altering circulating adipokine levels To increase the intra-abdominal mass of 'normal' fat tissue, epididymal fat pads were removed from a littermate donor and transplanted into the peritoneal cavity of the recipient. At the time mice were killed (10 weeks post-transplantation), transplanted fat pads were adherent to the peritoneum and were vascularised (Fig. 1a,b). Sham operations were performed in control littermates. Recipient mice tolerated the surgical procedure and the fat transplantation well, with no adverse events noted, and weight gain during the ensuing 10 weeks comparable to that of shamoperated littermate mice (Fig. 1c).

Recipient mice had a $50 \%$ increase in their combined (endogenous + transplanted) intra-abdominal perigonadal fat depot mass $(p<0.005$, Table 1$)$. Fat tissue morphometry was performed on histological sections stained with haematoxylin-eosin (Fig. 2a). Adipocyte cell perimeter in the transplanted and endogenous fat pads did not differ at the time of transplantation (transplanted fat pad: 4,853 \pm 976 [arbitrary units], recipient endogenous fat pad: 4,056 \pm 785 , sham-operated fat pad: 4,170 $\pm 798 ; p=0.72$ ANOVA). However, adipocytes of endogenous epididymal fat pads in both sham-operated and recipient mice hypertrophied from transplantation to time of killing (adipocyte perimeter increase $1.64 \pm 0.28$-fold, $p<0.05$ and $1.73 \pm 0.31$-fold, $p<0.05$, in sham-operated and recipient mice, respectively), whereas adipocyte size of the transplanted fat pad did not increase $(0.91 \pm 0.14$-fold, $p=0.47)$ (Fig. $2 b)$. The combined intra-abdominal fat depot (endogenous + transplanted) did not result in a significant difference in total body weight between the recipient and control groups (Table 1). Consistent with this observation, circulating levels of adiponectin, leptin, resistin and NEFA (Table 1) in recipients compared with sham-operated controls were not statistically different ( $p=0.12,0.15,0.59$ and 0.16 , respectively). Thus,

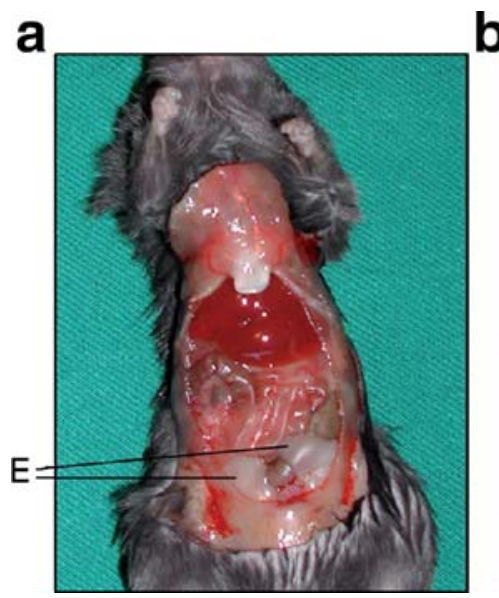

b

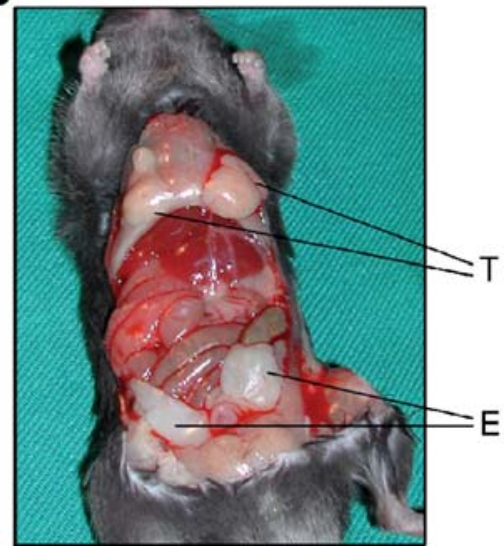

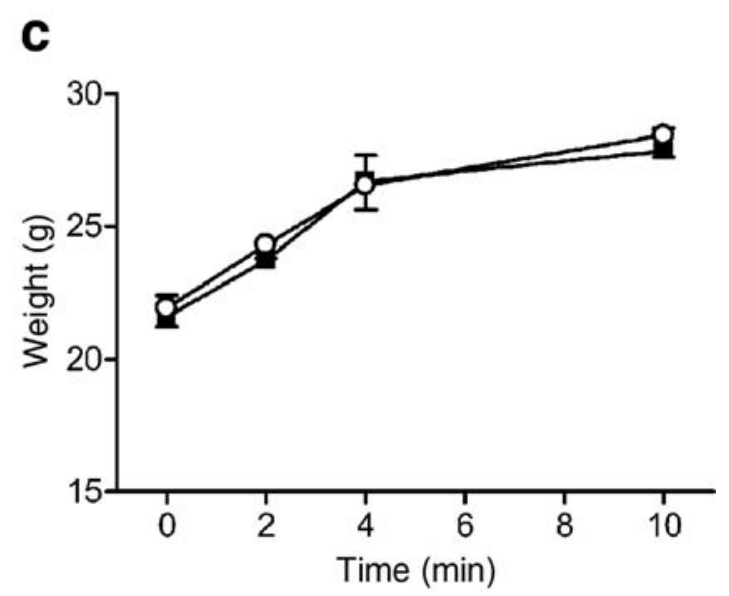

10 weeks post-surgery, the abdominal cavity was opened, and transplanted $(\mathrm{T})$ or endogenous (E) fat pads were visualised. a, sham; b, recipient. c Weight gain in recipient (open circles) and sham-operated littermate mice (closed squares). Results represent the mean $\pm \mathrm{SEM}$ of eight or nine animals per group
Fig. 1 Intra-abdominal transplanted fat is viable and weight gain is similar in sham-operated and recipient mice. a, b Six-week-old mice received intraperitoneal transplantation of two epididymal fat pads (approximately $175 \mathrm{mg}$ ) removed from a littermate donor, as detailed in the Materials and methods. Sham-operated mice served as controls. At 
Table 1 Total body weight, perigonadal fat pad weight and circulating plasma levels of adipokines and NEFA

\begin{tabular}{lccc}
\hline & Sham & Recipient & $p$ value \\
\hline Body weight $(\mathrm{g})$ & $28.0 \pm 0.7$ & $27.4 \pm 0.9$ & 0.42 \\
Fat depots & & & \\
$\quad$ Perigonadal depots, total $(\mathrm{mg})$ & $365 \pm 22$ & $508 \pm 23$ & $<0.005$ \\
Endogenous fat pad $(\mathrm{mg})$ & $365 \pm 22$ & $320 \pm 18$ & 0.32 \\
$\quad$ Transplanted fat pad (mg) & & $188 \pm 9$ & \\
Circulating adipokines & & & \\
Adiponectin $(\mu \mathrm{g} / \mathrm{ml})$ & $13.2 \pm 1.0$ & $11.1 \pm 0.8$ & 0.12 \\
Leptin $(\mathrm{pg} / \mathrm{ml})$ & $1,330 \pm 180$ & $1,075 \pm 71$ & 0.15 \\
Resistin $(\mathrm{pg} / \mathrm{ml})$ & $2,214 \pm 407$ & $1,912 \pm 421$ & 0.59 \\
NEFA $(\mathrm{mmol} / \mathrm{l})$ & $1.14 \pm 0.08$ & $1.01 \pm 0.04$ & 0.16 \\
\hline
\end{tabular}

transplanted fat increased intra-abdominal mass of adipose tissue while retaining adipocyte size of the transplanted fat, but it did not alter total body weight or circulating levels of secreted adipose tissue products.

Recipient mice with transplantation-mediated increase in intra-abdominal fat mass exhibit improved glucose tolerance in the fasting state We first assessed whether intraabdominal fat transplantation altered insulin sensitivity in the non-fasting state. For this, insulin sensitivity was measured 4 weeks after transplantation, after overnight feeding followed by $3 \mathrm{~h}$ of food withdrawal. Glucose lowering during post-absorptive ipITT was identical between recipient and sham-operated littermate mice (Fig. 3). To evaluate the effect of fat transplantation on glucose homeostasis in the fasting state, ipGTTs were performed. At 4 weeks post-transplantation, overnight-fasted mice exhibited improved glucose tolerance, as manifested by lower plasma glucose levels at 15, 30, 45, 60, 90 and
120 min after glucose administration, resulting in a $20 \%$ decrease in the AUC for glucose (recipient: $999 \pm 46$, shamoperated: $1267 \pm 66 ; p<0.001$, Fig. $4 \mathrm{a}, \mathrm{b})$. To test whether this effect was observable after a shorter fasting period, mice were fasted for $7 \mathrm{~h}$ prior to ipGTT. Glucose levels during ipGTT during these conditions were somewhat more variable (as evidenced by larger SEMs), but overall, recipient mice had lower glucose levels and lower mean glucose AUCs than sham-operated controls (Fig. 4c,d). Circulating insulin levels were also measured during ipGTT ( $7 \mathrm{~h}$ of fasting) and tended to be lower in the transplanted mice than in controls, particularly in the basal fasting state (baseline: $79.8 \pm 8.2$ vs $113.9 \pm 20.9 \mathrm{pmol} / 1, p=0.13 ; 15 \mathrm{~min}$ : $134.6 \pm 13.5$ vs $150.2 \pm 7.6 \mathrm{pmol} / 1, p=0.41 ; 30 \mathrm{~min}: 126.7 \pm$ 18.3 vs $139.8 \pm 19.3 \mathrm{pmol} / 1, p=0.62$ ). These results suggest that the post-challenge insulin secretory response was maintained (and not exaggerated) in mice receiving intraperitoneal fat transplantation, while insulin sensitivity in the fasting state was improved.

To further assess fasting insulin sensitivity, HOMA-IR was calculated. Fasting glucose levels were significantly $(p=0.03)$ lower in recipient mice (Fig. 5a), while insulin levels tended to be lower, although this difference did not reach statistical significance $(p=0.13$, Fig. $5 \mathrm{~b})$. Nevertheless, HOMA-IR was found to be statistically $(p=0.02)$ lower in recipient mice than in sham-operated controls (Fig. 5c), suggesting that an increase of intra-abdominal fat mass by transplantation of normal fat in fact improved fasting insulin sensitivity. To further verify this observation, we assessed whether there was a 'dose-effect' between the amount of transplanted fat and HOMA-IR. Consistent with the expected relationship between body mass and insulin sensitivity, total body weight at the time mice were killed positively correlated with HOMA-IR $(r=0.672, p=0.024)$
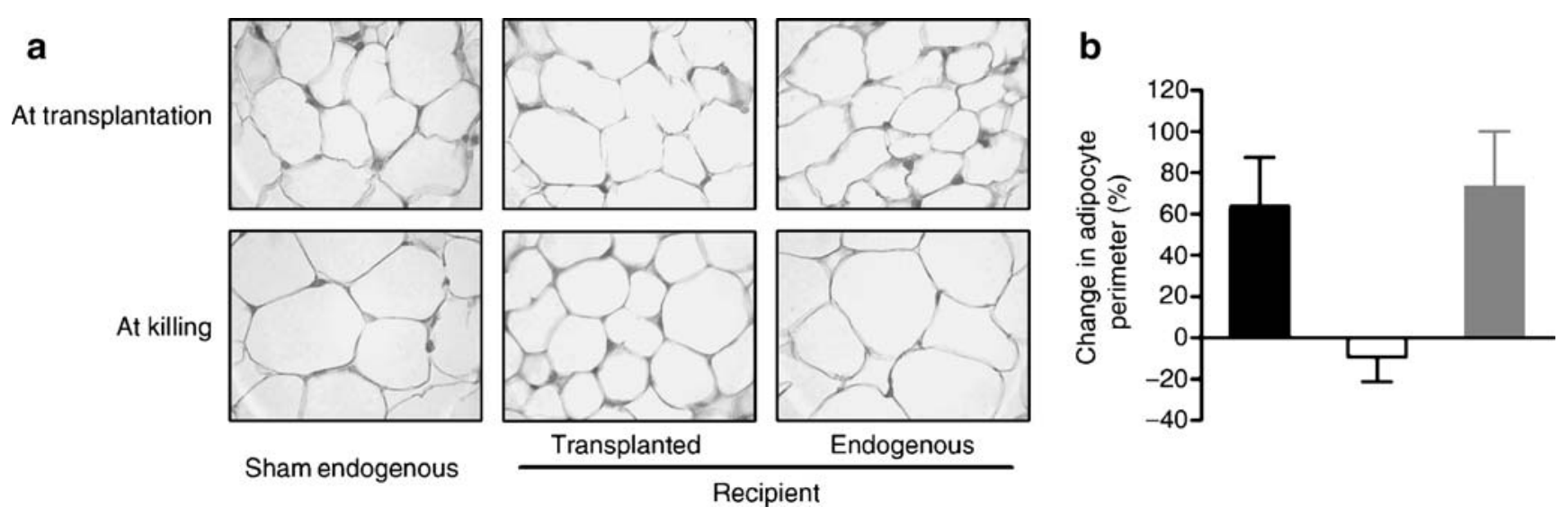

Fig. 2 Adipocyte size of transplanted fat pads remains stable over time. a Representative, haematoxylin- and eosin-stained histological sections of endogenous or transplanted fat pads from recipient or sham-operated mice at the time of transplantation or after killing (10 weeks later). Adipocyte cell perimeter was measured using ImageJ. b Up to 470 cells for each fat pad were analysed. The mean \pm SEM of four different mice is

depicted here. All values are expressed relative to the adipocyte cell perimeter of transplanted epididymal fat pad at the time of transplantation. Black bar, endogenous fat pad of sham-operated mice; white bar, transplanted fat pad of recipient mice; grey bar, endogenous fat pad of recipient mice 


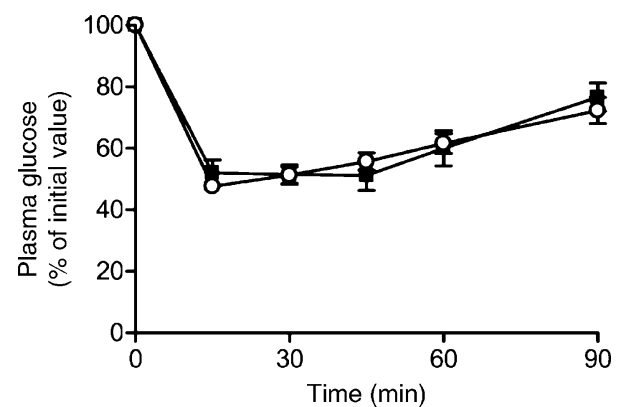

Fig. 3 Intra-abdominal transplantation of fat tissue does not alter post-absorptive insulin tolerance. Intraperitoneal ITT $(0.75 \mathrm{U} / \mathrm{kg}$ body weight) was performed 4 weeks after surgery in recipient (open circles) and sham-operated mice (filled squares) that had fasted for $3 \mathrm{~h}$. Blood was collected from the tail vein at the indicated time points. Results are the mean \pm SEM of nine or ten different animals in each group

by simple correlation analysis, whereas correlation between the transplanted fat weight and HOMA-IR tended to be negative $(r=-0.42, p=0.19)$ (Fig. 5d). Therefore, to evaluate the effect of fat transplantation independently of total body weight, we assessed the association between the transplanted fat weight and HOMA-IR, while adjusting for total body weight at time of death. Using a linear regression model, we found that the beta coefficient between the transplanted fat weight and HOMA-IR was $-0.505, p=$ 0.031 , while the $\beta$ coefficient in the same model for total body weight was $0.728, p=0.006$. This suggests that, independently of the positive relationship between
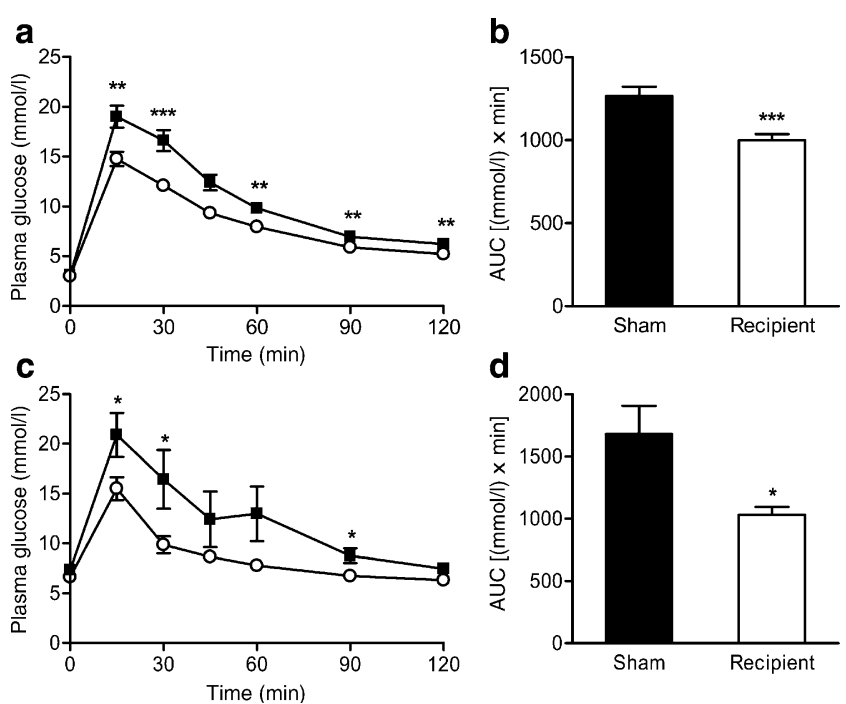

Fig. 4 Lean mice with transplantation-mediated increase in intraabdominal fat mass exhibit improved fasting glucose tolerance. Intraperitoneal GTT ( $2 \mathrm{~g}$ glucose/kg body weight) was performed 4 weeks after surgical procedure in recipient (open circles) and shamoperated mice (filled squares) that had fasted overnight (a, b) or for only $7 \mathrm{~h}$ (c, d). Results are expressed as mean plasma glucose concentration or $\mathrm{AUC} \pm \mathrm{SEM}$ from 15 (sham) or 16 (recipient) animals (a, b) or from six (sham) or seven (recipient) animals (c, d). ${ }^{*} p<0.05$, $* * p<0.01, * * * p<0.001$ (Student's $t$ test)
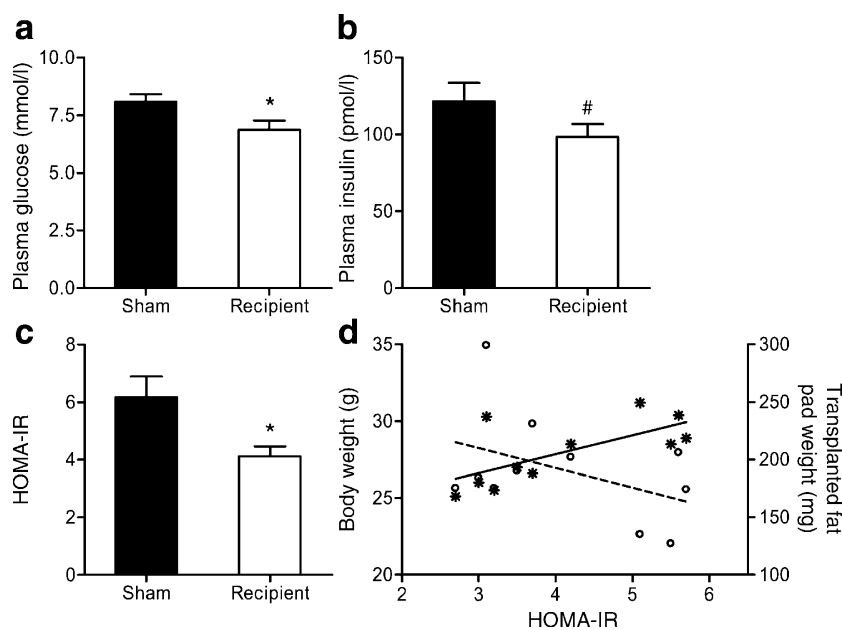

Fig. 5 The effect of fat transplantation on fasting glucose, insulin and HOMA-IR. Fasting plasma glucose (a) and insulin (b) were measured in recipient and sham-operated mice after $7 \mathrm{~h}$ of food withdrawal, and HOMA-IR (c) was calculated as detailed in the Methods section. Results represent the mean \pm SEM of 11 to 12 different animals per group. d Pearson correlation between HOMA-IR and total body weight (stars, continuous line $)(r=0.672, p=0.024)$ or transplanted fat pad weight (open circles, dotted line) $(r=-0.42, p=0.19)$ was performed. ${ }^{*} p<0.05 ; \# p=0.13$

HOMA-IR and total body weight, increasing the amount of transplanted fat predicts increased fasting insulin sensitivity.

\section{Discussion}

It is unclear whether increased intra-abdominal fat mass as such or altered fat tissue biology contributes to the metabolic derangement associated with obesity. The first notion is supported by findings that intra-abdominal fat mass reduction improves insulin sensitivity in rats [4], whereas implantation of cultured adipocytes into the peritoneal cavity of mice induces insulin resistance [11]. Here we used intra-abdominal fat tissue transplantation to increase fat mass. Surprisingly, lean recipients of epididymal fat transplant from lean littermates exhibited improved fasting glucose tolerance and increased insulin sensitivity. Thus, increasing intra-abdominal fat mass by transplantation of normal adipose tissue did not mimic the effects of increased fat mass in obesity.

In this model, fat mass of transplanted fat tissue was inversely correlated with HOMA-IR, independently of the expected positive association between total body weight and HOMA-IR. Histological examination of the transplanted adipose tissue revealed that after 10 weeks of observation (transplantation to scarification) adipocyte cell size increased significantly in endogenous fat pads, whereas it remained unchanged in the transplanted fat pads 
(Fig. 2b). Insulin action was previously shown to be dependent on fat cell size: large adipocytes correlate well with insulin resistance [12-14] and show decreased insulinstimulated glucose uptake [15]. Interestingly, the transplanted fat depot exhibited features reminiscent of those induced in adipose tissue by thiazolidinediones. These drugs were shown to increase cellularity in adipose tissue by promoting fat cell differentiation and inducing apoptosis of large adipocytes, resulting in smaller, more insulinsensitive adipocytes that secrete higher levels of adiponectin $[16,17]$. It is thus possible that the insulin-sensitising effects of the transplant are secondary to similar alterations in the transplanted adipose tissue. We therefore assessed whether alterations in circulating levels of secretory products of fat tissue mediated the improved glucose tolerance and increased insulin sensitivity in mice receiving fat transplantation. However, we did not observe any significant increases in the circulating levels of potential insulin-sensitising adipokines like leptin or adiponectin [18] or any significant decreases in factors associated with reduced insulin sensitivity (resistin and NEFA, Table 1).

Although potentially informative, such analysis is limited in at least two major aspects. First, we measured total adiponectin levels, but recent studies suggest that the circulating high-molecular-mass complex of adiponectin correlates better with insulin sensitivity than total serum adiponectin levels $[19,20]$. Second, with regard to the unaltered circulating levels of adipokines and NEFA observed by us, it should be noted that venous drainage of intra-abdominal fat is to the portal system. It therefore remains possible that intra-portal levels of secreted products were affected, thereby predominantly influencing liver glucose homeostasis. Such a scenario, in which the greatest effect of intra-abdominal fat tissue transplantation is seen in the liver, is also supported by the fact that the insulinsensitising effects of intra-abdominal fat transplantation was largely observed in the fasting state, conditions in which glycaemia is largely controlled by hepatic glucose production and insulin sensitivity.

In conclusion, our findings suggest that in addition to increased fat mass, altered adipose tissue function is required to reproduce obesity-related metabolic changes such as insulin resistance. These changes may include impaired metabolic function of adipocytes, changes in cellular composition of adipose tissue (e.g. macrophage infiltration) [21] and/or alterations in the secretory function of adipose tissue (inflammatory cytokines, adipokines, NEFA). The second possible conclusion is that augmentation of normally functioning adipose tissue appears to result in a new metabolic steady state with increased insulin sensitivity. Such compensation and adaptation mechanisms may be unable to counterbalance larger degrees of fat tissue expansion. Yet, the results suggest that multiple factors in the cross-talk mechanisms between different insulin-sensitive tissues need to be affected in order to impair glucose homeostasis in obesity. The notion that adipose tissue function (rather than mass) and its inter-organ cross-talk are key determinants of obesity-induced metabolic derangement further defines this disorder as an endocrinopathy, and adipose tissue as an endocrine organ.

Acknowledgements This work was supported by grants from the Medical Faculty of the University of Zurich and the Swiss National Science Foundation grant no. 310000-112275 (to D. Konrad). We would like to greatly acknowledge I. Shai from the S. Daniel Abraham International Center for Health and Nutrition, Ben-Gurion University for valuable input and guidance in the statistical analysis of our data and M. Arras for her expert veterinary advice.

Duality of interest No conflict of interest existed for any of the authors.

\section{References}

1. Das M, Gabriely I, Barzilai N (2004) Caloric restriction, body fat and ageing in experimental models. Obes Rev 5:13-19

2. Van Gaal LF, Mertens IL, Abrams PJ (2003) Health risks of lipodystrophy and abdominal fat accumulation: therapeutic possibilities with leptin and human growth hormone. Growth Horm IGF Res 13(Suppl A):S4-S9

3. Thorne A, Lonnqvist F, Apelman J, Hellers G, Arner P (2002) A pilot study of long-term effects of a novel obesity treatment: omentectomy in connection with adjustable gastric banding. Int $\mathrm{J}$ Obes Relat Metab Disord 26:193-199

4. Barzilai N, She L, Liu BQ et al (1999) Surgical removal of visceral fat reverses hepatic insulin resistance. Diabetes 48:94-98

5. Klein S, Fontana L, Young VL et al (2004) Absence of an effect of liposuction on insulin action and risk factors for coronary heart disease. N Engl J Med 350:2549-2557

6. Raz I, Eldor R, Cernea S, Shafrir E (2005) Diabetes: insulin resistance and derangements in lipid metabolism. Cure through intervention in fat transport and storage. Diabetes Metab Res Rev 21:3-14

7. Cancello R, Clement K (2006) Is obesity an inflammatory illness? Role of low-grade inflammation and macrophage infiltration in human white adipose tissue. BJOG 113:1141-1147

8. Konrad D, Bilan PJ, Nawaz Z et al (2002) Need for GLUT4 activation to reach maximum effect of insulin-mediated glucose uptake in brown adipocytes isolated from GLUT4myc-expressing mice. Diabetes 51:2719-2726

9. Xia Z, Sniderman AD, Cianflone K (2002) Acylation-stimulating protein (ASP) deficiency induces obesity resistance and increased energy expenditure in ob/ob mice. J Biol Chem 277:45874-45879

10. Haluzik MM, Lacinova Z, Dolinkova M et al (2006) Improvement of insulin sensitivity after peroxisome proliferator-activated receptor-alpha agonist treatment is accompanied by paradoxical increase of circulating resistin levels. Endocrinology 147:4517-4524

11. Shibasaki M, Takahashi K, Itou T et al (2002) Alterations of insulin sensitivity by the implantation of 3T3-L1 cells in nude mice. A role for TNF-alpha? Diabetologia 45:518-526

12. Weyer C, Foley JE, Bogardus C, Tataranni PA, Pratley RE (2000) Enlarged subcutaneous abdominal adipocyte size, but not obesity itself, predicts type II diabetes independent of insulin resistance. Diabetologia 43:1498-1506 
13. Schneider BS, Faust IM, Hemmes R, Hirsch J (1981) Effects of altered adipose tissue morphology on plasma insulin levels in the rat. Am J Physiol 240:E358-E362

14. Smith U (1970) Insulin responsiveness and lipid synthesis in human fat cells of different sizes: effect of the incubation medium. Biochim Biophys Acta 218:417-423

15. Czech MP (1976) Cellular basis of insulin insensitivity in large rat adipocytes. J Clin Invest 57:1523-1532

16. Okuno A, Tamemoto H, Tobe K et al (1998) Troglitazone increases the number of small adipocytes without the change of white adipose tissue mass in obese Zucker rats. J Clin Invest 101:1354-1361

17. de Souza CJ, Eckhardt M, Gagen K et al (2001) Effects of pioglitazone on adipose tissue remodeling within the setting of obesity and insulin resistance. Diabetes 50:1863-1871
18. Niswender KD, Schwartz MW (2003) Insulin and leptin revisited: adiposity signals with overlapping physiological and intracellular signaling capabilities. Front Neuroendocrinol 24:1-10

19. Fisher FF, Trujillo ME, Hanif W et al (2005) Serum high molecular weight complex of adiponectin correlates better with glucose tolerance than total serum adiponectin in Indo-Asian males. Diabetologia 48:1084-1087

20. Waki H, Yamauchi T, Kamon J et al (2003) Impaired multimerization of human adiponectin mutants associated with diabetes. Molecular structure and multimer formation of adiponectin. J Biol Chem 278:40352-40363

21. Wellen KE, Hotamisligil GS (2005) Inflammation, stress, and diabetes. J Clin Invest 115:1111-1119 